TITLE:

\title{
Molecular dynamics study of the angular dependence of reactive cluster impacts
}

$\operatorname{AUTHOR}(S):$

Aoki, Takaaki; Matsuo, Jiro

\section{CITATION:}

Aoki, Takaaki ...[et al]. Molecular dynamics study of the angular dependence of reactive cluster impacts. Nuclear Instruments and Methods in Physics Research Section B: Beam Interactions with Materials and Atoms 2005, 241(1-4): 594-598

ISSUE DATE:

2005-12

URL:

http://hdl.handle.net/2433/8931

\section{RIGHT:}

この論文は出版社版でありません。引用の際には出版社版をご確認ご 利用ください。; This is not the published version. Please cite only the published version. 
Molecular Dynamics Study of the Angular Dependence of Reactive Cluster Impacts

${ }^{*}$ Takaaki Aoki ${ }^{1,2)}$ and Jiro Matsuo ${ }^{2)}$

1) Collaborative Research Center for Advanced Quantum Beam Process Technology

2) Quantum Science and Engineering Center, Kyoto University

\section{ABSTRACT}

The collisional processes of fluorine clusters impacting at various incident angles on bare silicon surfaces were studied, and the change of surface profile, sticking probabilities of $\mathrm{F}$ atoms, sputtering yield and the distribution of the sputtered particles were examined. When a $\left(\mathrm{F}_{2}\right)_{300}$ cluster with $10 \mathrm{eV} /$ atom impacts on a $\mathrm{Si}(100)$ surface at normal incident, the cluster penetrates the surface causing a spherical crater structure with the $\mathrm{F}$ atoms densely covered. As the incident angle increases, the penetration depth of cluster decreases and the simulation shows an asymmetric crater profile. Especially at an incident angle of 75 degrees, the surface profile did not change but $\mathrm{F}$ atoms were deposited over a wide area. The distribution of sputtered particles also depends on incident angle. At normal incidence, the $\mathrm{Si}$ atoms are sputtered as well as silicon-fluoride molecules such as $\mathrm{SiF}$ and $\mathrm{SiF}_{2}$, while only $\mathrm{SiF}_{x}$ molecules are sputtered at 75 degrees of incident angle. From these results the surface etching process caused by reactive cluster impacts are discussed.

PACS: 36.40.-c (Atomic and molecular clusters), 83.10.Rs (Computer simulations of molecular and particle dynamics)

KEYWORDS: gas cluster ion beam, molecular dynamics, sputtering, fluorine, silicon

\footnotetext{
* Contact author:

Takaaki Aoki

Quantum Science and Engineering Center, Gokasho, Uji, Kyoto, 611-0011, JAPAN

TEL:+81-774-38-3977 / FAX:+81-774-38-3978 / E-MAIL: aoki@sakura.nucleng.kyoto-u.ac.jp
} 


\section{INTRODUCTION}

The impact processes of gas clusters on solid surfaces are of great interest for new surface modification techniques [1,2]. A Cluster is an aggregated material, which consists of several tens to thousands atoms. When a cluster is accelerated and impacts on a solid target, high-density atomic collisions occur and the kinetic energy of the cluster is deposited in very shallow area of the target. This collision process is different from that of monomer ions, and is the basis of a new surface modification technology. The study of gas cluster ion impact began mainly with argon gas clusters, which is an inert material and is suitable for understanding the physical effect of the multiple collision effect of clusters. Recently, it has been reported that, gas cluster ion beams can be generated with various gas-phase materials such as $\mathrm{SF}_{6}, \mathrm{CF}_{4}$, etc [3]. These new source materials allow gas cluster ion beam surface modification processes that combine the non-linear effects inherent in cluster impacts with the chemical properties of source gas materials. For example, in the case of sputtering, it has been demonstrated that with $\left(\mathrm{SF}_{6}\right)_{2000}$ clusters irradiating $\mathrm{Si}$ or $\mathrm{W}$ substrates, the sputtering yield is 10 times higher than that of $A_{2000}$ clusters, which in turn is 10 times higher than that of monomer ions with the same total acceleration energy of $20 \mathrm{keV} \mathrm{[4].} \mathrm{The} \mathrm{high}$ etching rate of reactive cluster ions is now studied for application in industrial precision nano-scale patterning processes [5]. It is important to understand the mechanism of the collisional and etching processes of reactive clusters in order to optimize the surface process for specific industrial requirements. In this study, molecular dynamics (MD) simulations of fluorine clusters impacting on bare silicon surface were performed. The angular dependence of the change of surface profile, the sticking of $\mathrm{F}$ atoms and the sputtering of $\mathrm{Si}$ atoms are examined. The results of the etching process by reactive cluster ion are discussed. 


\section{SIMULATION MODEL}

Our group has studied the collisional processes of fluorine atoms, molecules and clusters on silicon targets using the molecular dynamics method [6,7]. In this work, the angular dependence of the collisional process of fluorine clusters is reported. In order to describe the interactions between Si-Si, Si-F and F-F, the Stillinger and Weber (SW) type potential model was applied. The SW type potentials were developed by Stillinger et al. [8-10] and modified by Weaklime et al. [11]. This potential model was modeled from the results of ab-initio calculations and well represents the energy and structure of silicon-fluoride molecules and surface sticking processes $A n\left(\mathrm{~F}_{2}\right)_{300}$ cluster was used as a projectile; it was annealed in the simulation at $10 \mathrm{~K}$ before impact using the Langevine dynamics method. After annealing, the fluorine cluster has a spherical and amorphous structure, which avoids artifacts due to the structure of the cluster.

The $\operatorname{Si}(001)$ substrate was prepared as the target. The target consists of about 500,000 atoms and its dimension is $360 \AA \times 360 \AA \times 100 \AA$, which is large enough to receive the incident energy when the $\mathrm{F}$ atoms collide at glancing angle. A periodic boundary condition is applied to the horizontal plane. The Si atoms in the lower four layers are fixed to keep the bulk status and the bottom region of the substrate (up to $1 / 4$ the thickness) is simulated by the Langevine dynamics method to keep the substrate temperature at $300 \mathrm{~K}$. It should be noted that, the starting target material in this work is bare silicon, so that these simulations represent the very initial stage of reactive cluster ion impact, not the steady state expected for real experiments, where the surface is covered with silicon fluoride compounds.

\section{RESULTS AND DISCUSSION}

The $\left(\mathrm{F}_{2}\right)_{300}$ cluster was given a total energy of $6 \mathrm{keV}$ and irradiated at various incident angles on the $\mathrm{Si}(100)$ target. Each $\mathrm{F}$ atom in the cluster shares the total incident energy, so each $\mathrm{F}$ 
atom carries $10 \mathrm{eV}$. Fig. 1 shows snapshots of the impact processes of a fluorine cluster. In Fig. 1 black and gray circles indicate $\mathrm{F}$ and $\mathrm{Si}$ atoms, respectively. When the cluster impact is normal to the surface, the cluster penetrates into the target. During the collisional process, large numbers of collisions occur between the cluster and the substrate atoms. Because of this multiple collision effect, the incident atoms are directed not only vertically but also horizontally, which results in a hemispherical crater formation on the target surface. After the impact, some fluorine atoms remain stuck on the surface while others desorb into the vacuum. As the incident angle increases, the penetration depth of the cluster becomes shallower, and the incident $\mathrm{F}$ atoms tend to evaporate in different directions from that of the incident cluster. At an incident angle of 75 degrees, the incident cluster slides on the target surface with its upper part remaining in the cluster state. After several picoseconds, the cluster breaks up and many $\mathrm{F}$ atoms leave the target.

Fig. 2 shows the surface profiles of the targets at 16ps after F clusters impact with various incident angles. As the incident angle increases, the depths of the craters decrease and the shapes become asymmetric. Finally, at 75 degrees of the incident angle, the crater-like shape does not occur. The previous work on Ar clusters impacting on Si targets [12] has shown that when a cluster impacts in the normal direction, there is threshold energy of several $\mathrm{eV}$ per atom where the cluster will penetrate the surface of the target and create crater-like damage. For this study, such a threshold energy would as apply to the vertical component of the incident energy, which decreases with a $\cos ^{2} \theta$ law. This value is calculated to be about $0.67 \mathrm{eV} /$ atom at 75 degrees and $2.5 \mathrm{eV} /$ atom at 60 degrees. The rapid decrease of the vertical component of incident energy causes the difference in the profiles in Fig. 2.

Fig. 3 shows the angular dependence of the fluorine sticking probability for a $\left(\mathrm{F}_{2}\right)_{300}$ cluster with $10 \mathrm{eV} /$ atom. It is natural that the sticking probability depends on the number of 
adsorption sites on the surface. For glancing angle ion irradiation, the sticking probability should increase with incident angle because the projection area of the cluster obeys $1 / \cos \theta$. However, Fig. 3 shows the sticking probability decreases as incident angle increases. Two reasons are considered. The first is that, when a $\left(\mathrm{F}_{2}\right)_{300}$ cluster impacts at a very large incident angle, some part of $\mathrm{F}$ atoms in the cluster do not contact the silicon target, as shown in the bottom line of Fig. 1. In other words, too many $\mathrm{F}$ atoms are supplied at the impact. Another reason is that, when the incident angle is small (very near to the normal), the cluster can penetrate the surface and make a crater, which increases the number of adsorption sites. From Fig. 3, at normal incidence, more than $300 \mathrm{~F}$ atoms remain within an area of about $100 \AA \times 100 \AA$ on the target surface, which implies that precursors can be formed at very high density with only one impact of the cluster and suggests an enhancement of the chemical reaction between fluorine and silicon atoms.

As can seen in Fig. 1, not only fluorine but also silicon atoms evaporate at all incident angles, and some sputtered atoms has molecule state. Fig. 4 shows the distribution of sputtered particles. In this study, the sputtered molecule was defined as a cluster in which atoms are linked with each other within $3.8 \AA$ of interatomic distance, but are not linked with substrate atoms at $16 \mathrm{ps}$ after impact. Therefore, it is noted that Fig. 4 eliminates the long-term thermal desorption process and only represents the sputtering process under high-density energy and momentum deposition in very short time range. At $0^{\circ}$ and $45^{\circ}$ incident angles, non-fluoridated silicon was observed as sputtered material as well as silicon-fluoride molecules such as $\mathrm{SiF}$ and $\mathrm{SiF}_{2}$. Under these collisional conditions, many $\mathrm{Si}$ atoms are moved horizontally along the surface, due to the multiple-collision process. During the multiple collisions, some target atoms leave from the edge of crater. This lateral sputtering effect is an important collisional process in large cluster impacts and leads to surface smoothing [13]. On the other hand, when the incident angle is as large as 75 degrees, most 
of sputtered particles are silicon-fluoride molecules. In this case, an enhanced chemical reaction due to the high-density irradiation effect is the dominant mechanism of sputtering. As for the total desorption yield, the maximum for both $\mathrm{Si}$ and $\mathrm{F}$ occurs at 45 degrees. In order to achieve higher etching, both the formation and desorption of the precursors should be enhanced. The results graphed in Fig. 4 indicate that at $45^{\circ}$ incident angle, crater formation contributes to the former enhancement mechanism and asymmetric momentum transfer due to glancing angle irradiation contributes to the latter one.

\section{CONCLUSIONS}

Molecular dynamics simulations of fluorine cluster impacts were performed in order to examine the surface etching process by reactive gas cluster impacts. It was shown that, by changing the incident angle, the shape of crater could be controlled. Additionally, when the vertical component of the incident energy is less than several $\mathrm{eV}$, a cluster cannot penetrate the target surface and chemical reaction only occur very near to the surface. The predicted sticking probability and sputter rate for $\left(\mathrm{F}_{2}\right)_{300}$ clusters with $6 \mathrm{keV}$ are large enough to form a high density of silicon-fluoride precursors on the target surface. The study of the sputtered products indicates that the sputtering process can be enhanced due to both the physical and the chemical processes of the cluster impact. The former mechanism is the horizontal momentum transfer effect termed as lateral sputtering and the latter is the high-density particle irradiation effect. These collisional processes occur in the region very near to the surface, shallower than $100 \AA$, so it is expected that the reactive gas cluster ion beam technique will achieve high efficiency with low damage when used as a nano-scale etching processes.

\section{ACKNOWLEDGEMENT}


This study is supported by Ministry of Economy, Trade and Industry (METI) and New Energy and Industrial Development Organization (NEDO) in Japan.

\section{REFERENCES}

[1] I. Yamada, J. Matsuo, N. Toyoda, T. Aoki, E. Jones and Z. Insepov, Mater. Sci. and Eng., A253 (1998) 249

[2] I. Yamada, J. Matsuo, Z. Insepov, T. Aoki, T. Seki and N. Toyoda, Nucl. Instr. and Meth.

B, 164-165 (2000) 949

[3] A. Kirkpatrick, Nucl. Instr. and Meth. B 206 (2003) 830.

[4] N. Toyoda, H. Kitani, N. Hagiwara, J. Matsuo and I. Yamada, Mater. Chem. and Phys., 54 (1998) 106

[5] E. Bourelle, A. Suzuki, A. Sato, T. Seki and J. Matsuo, Jpn. J. Appl. Phys., 43 (2004), L1253.

[6] T. Aoki, J. Matsuo and I. Yamada, Nucl. Instr. and Meth. B, 164-165 (2000) 546

[7] T. Aoki, J. Matsuo and I Yamada, Nucl. Instr. and Meth. B, 180 (2001) 164.

[8] F. H. Stillinger and T. A. Weber, J. Chem. Phys., 88 (1988) 5123

[9] F. H. Stillinger and T. A. Weber, Phys. Rev. Lett., 62 (1989) 2144

[10] T. A. Weber and F. H. Stillinger, J. Chem. Phys., 92 (1990) 6239

[11] P. C. Weakliem, C. J. Wu and E. A. Carter, Phys. Rev. Lett., 69 (1992) 200

[12] T. Aoki, J. Matsuo and G. Takaoka, Nucl. Instr. and Meth. B, 202 (2003) 278.

[13] T. Aoki, J. Matsuo, Z Insepov and I. Yamada, Nucl. Instr. and Meth. B, 121 (1997) 49. 


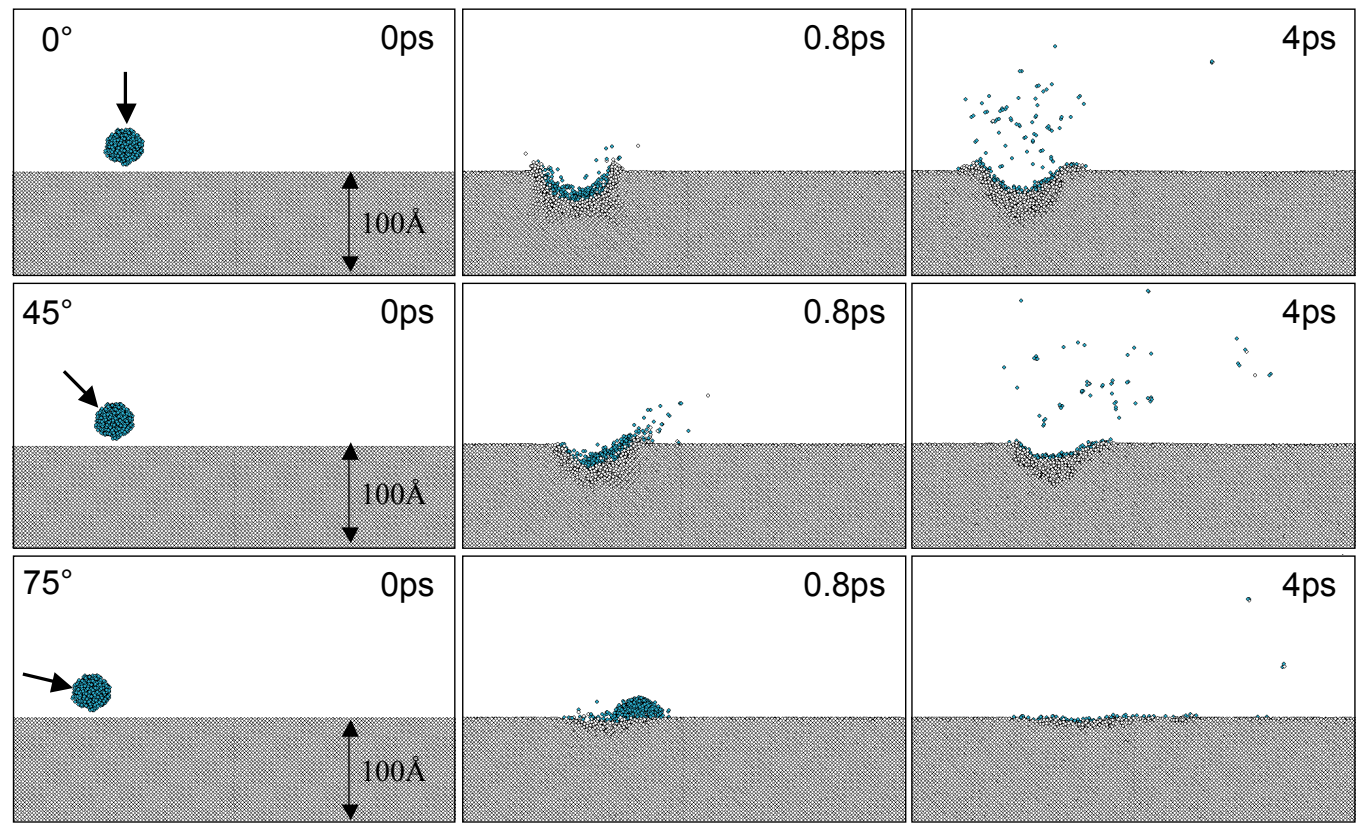

Fig. 1: snapshots of $\left(\mathrm{F}_{2}\right)_{300}$ clusters with $10 \mathrm{eV} /$ atom impacting on bare $\mathrm{Si}(100)$ surface at different incident angles.

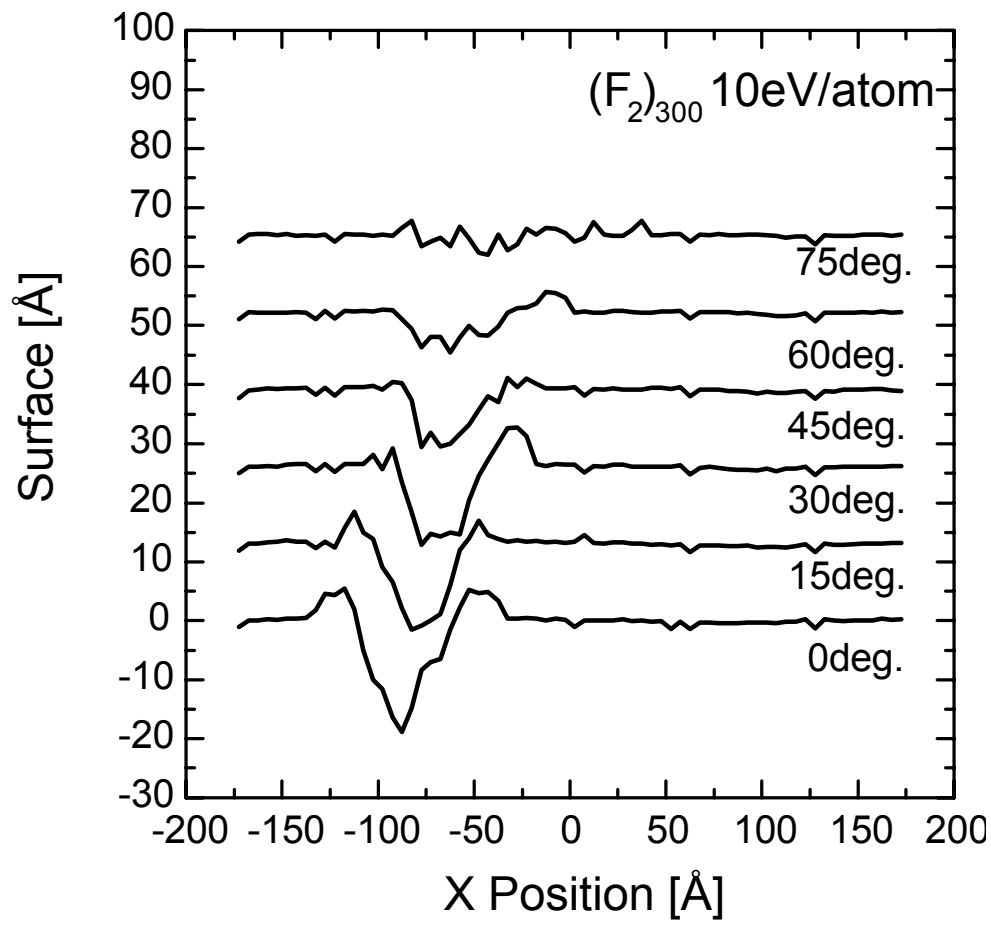

Fig. 2: surface profiles after impact by $\left(\mathrm{F}_{2}\right)_{300}$ clusters at different angles, 16ps after impact. 


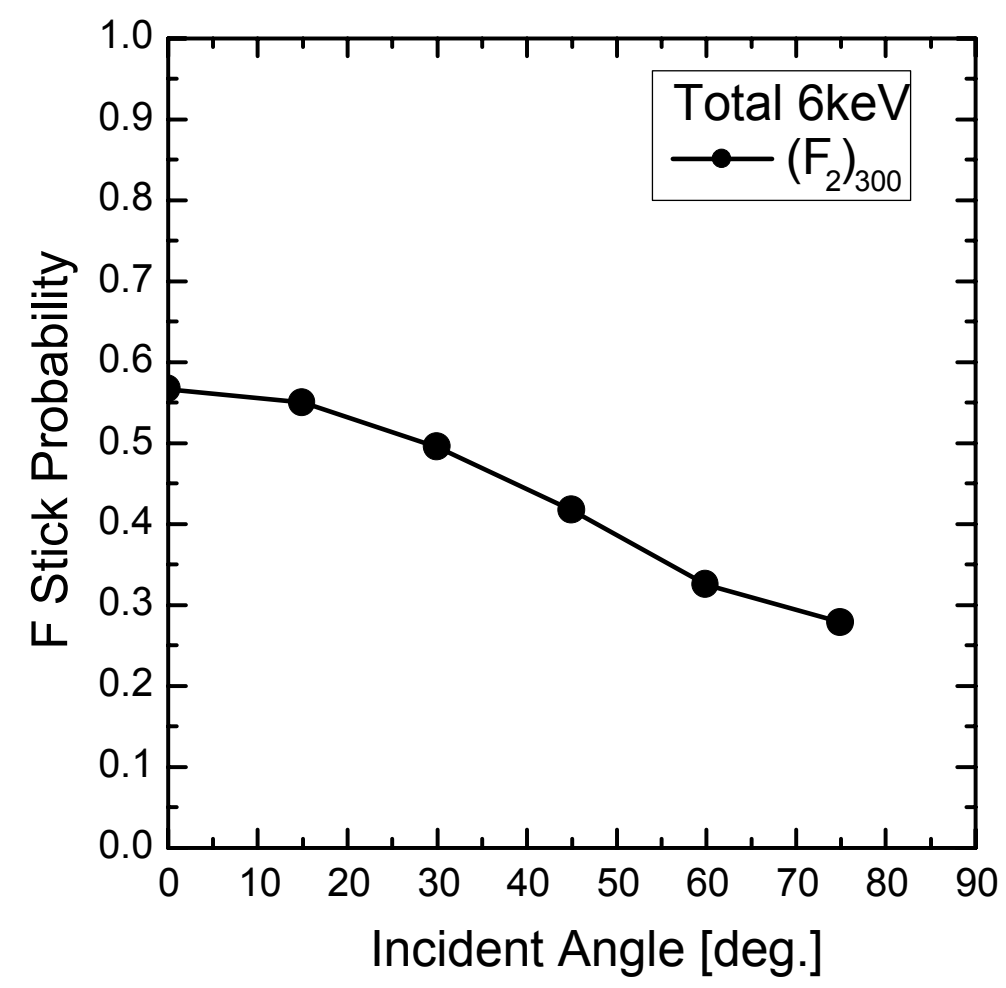

Fig.3: Incident angle dependence of the sticking probability of $\mathrm{F}$ atoms, 16ps after impact.
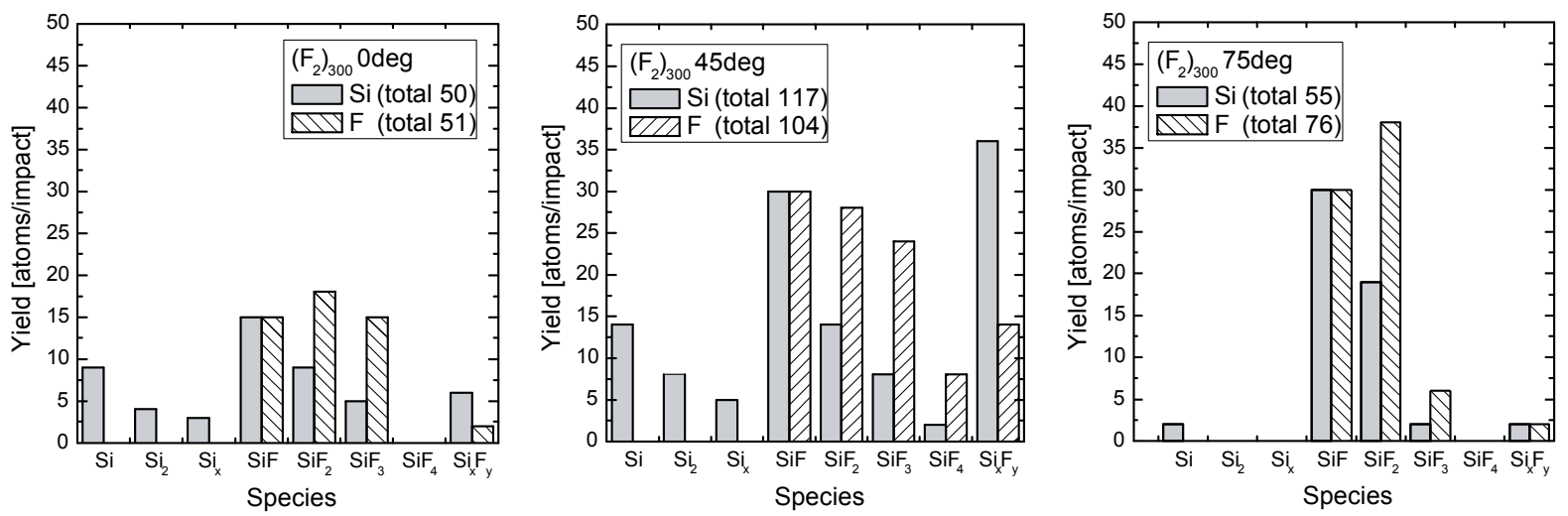

Fig. 4: Distributions of sputtered particle species caused by the impact of $\left(\mathrm{F}_{2}\right)_{300}$ clusters at 0 , 45 and 75 degrees of incident angle, 16ps after impact. 